\title{
The Art of Oncoimmunovaccinomics
}

\author{
Taruna Ikrar ${ }^{1,2,3 \#^{*}}$, Dito Anurogo ${ }^{4,5 \#}$ \\ ${ }^{1}$ Chairman of Medical Council, The Indonesian Medical Council, Jakarta \\ ${ }^{2}$ Aivita Biomedical Inc., Irvine, California \\ ${ }^{3}$ Department of Pharmacology, Faculty of Medicine, Malahayati University, Bandar Lampung \\ ${ }^{4}$ International PhD Program for Cell Therapy and Regeneration Medicine (IPCTRM), College of Medicine, Taipei Medical \\ University (TMU), Taipei City \\ ${ }^{5}$ Faculty of Medicine and Health Sciences, Universitas Muhammadiyah Makassar, Makassar \\ Email: *tarunaikrar@kki.go.id
}

How to cite this paper: Ikrar, T. and Anurogo, D. (2021) The Art of Oncoimmunovaccinomics. World Journal of Vaccines, 11 , 50-66.

https://doi.org/10.4236/wjv.2021.114007

Received: June 12, 2021

Accepted: November 22, 2021

Published: November 25, 2021

Copyright $\odot 2021$ by author(s) and Scientific Research Publishing Inc. This work is licensed under the Creative Commons Attribution International License (CC BY 4.0).

http://creativecommons.org/licenses/by/4.0/

\begin{abstract}
The art of oncoimmunovaccinomics will be concisely explained herein. This scientific insight will explore its history cancer immunotherapy, the molecular basis of cancer immunotherapy, cancer immunotherapeutic approach, cancer immunosurveillance and immunoediting, the hallmarks of cancer (can be) revisited, nanotechnology-based cancer immunotherapy, immunovaccinomics, vaccinomics approach, the theory of vaccinology, adversomics, and immunogenomic perspectives.
\end{abstract}

\section{Keywords}

Oncology, Cancer, Immunotherapy, Nanoparticles, Immunovaccinomics, Vaccinology, Adversomics

\section{Introduction}

There have been different cancer treatment modalities for a long time. Surgery, radiation, chemotherapy, targeted medications, and immunotherapy are the treatments available. Firstly, let us begin with a discussion of surgery. Surgery has been used to excise active tumor cells to avoid their development and spread since the 1800s. Even so, surgery has its drawbacks, such as the ineligibility of certain inaccessible tumors and the reduced efficacy of surgery if the tumor has already spread.

Secondly, let us reveal about radiation in a nutshell. Since the early 1900s, radiation has been used to destroy cancerous cells by using extremely focused $\mathrm{X}$-rays or radioactive isotopes. Nevertheless, radiation has its restriction. It pos${ }^{\#}$ These authors (T.I. \& D.A.) contributed equally to this work.

${ }^{\star}$ Corresponding author. 
sesses limited efficacy if tumor has already started to spread; in addition, it can be potentially harmful for vital organs juxtaposed tumors.

Thirdly, let us provide a brief overview of chemotherapy. Since the late 1940's, cytotoxic drugs have been used to destroy and suppress cancer cells. However, it has its drawback, such as high toxicity and limited efficacy in killing the entire tumor, leading to high rates of recurrence. Capecitabine, vinorelbine, cyclophosphamide, and other chemotherapy drugs are examples.

Fourthly, there's the issue of targeted medications. Since the 2000s, these approaches have been thought to be interfering with a process that is necessary for, or supports, tumor development. Nonetheless, it has limitations, particularly in terms of the tumor types that are eligible; high efficiency but short durability leads to high recurrence rates. Imatinib and Lapatinib are two examples of molecular targeted therapy. Parts of them are monoclonal antibodies, for example: Trastuzumab, Rituximab, Alemtuzumab, Bevacizumab, Cetuximab. Some of them are immunoconjugates, such as: Gemtuzumab, Ibritumomab tiuxetan, Tositumomab [1].

Finally, we have immunotherapy. It has been recognized as supporting the immune system's innate ability to recognize and eliminate tumor cells since the 2010s. Immunotherapy has a number of advantages, including the fact that it can be used at any stage of disease, even metastatic tumors; responses are longlasting; it has lower toxicity profiles; and synergistic effects with other treatments.

\section{Cancer Immunotherapy}

The fundamental concept of cancer immunotherapy is utilizing the body's immune system to battle cancer. Cancer immunotherapy is classified into two groups, namely active and passive (adoptive). It is called active if the host immune system is stimulated, whereas passive immunotherapy is when there is a transfer of effector molecules or cells (antibody, CTL or Cytotoxic T lymphocytes) to the patient. Both active and passive divided by 2 types are specific and nonspecific, it explained in Table 1.

Over the last three decades, several anticancer immunotherapeutic have been created. They are immunostimulatory cytokines; immunogenic cell death inducers; tumor-targeting and immunomodulatory monoclonal antibodies (mAbs); oncolytic viruses; adoptive cell transfer; pattern recognition receptor (PRR) agonists; peptide dendritic cell (DC)-, and DNA-based anticancer vaccines; and inhibitors of immunosuppressive metabolism (Figure 1) [2].

Concisely, there are five ways to combat cancer, i.e., monoclonal Ab (tumor specific Ab: herceptin), checkpoint inhibitors (anti-CTLA-4 Ab: ipilimumab, anti-PD 1 Ab: Nivolumab and pembrolizumab), cancer vaccine (provenge as first cancer treatment vaccine), adoptive cell therapy (CART: Chimeric Antigen Receptor-Modified T Cells), and oncolytic viruses (Talimogene laherparepvec TVEC). 
Table 1. Classification of cancer immunotherapy (Adapted from [2]).

\begin{tabular}{lll}
\hline & Specific & Nonspecific \\
\hline Active & Cancer vaccines & Interferon alpha \\
& Tumor cell vaccines & Interleukin 2 \\
Tumor lysates/oncolysates & Tumor necrosis factor alpha \\
& Recombinant viral vaccines & Adjuvants \\
& Peptide vaccines & Bacille Calmette-Guerin \\
& Naked DNA/RNA & Corynebacterium parvum \\
& DC vaccines & Detox \\
& & Coley's toxin \\
\hline $\begin{array}{l}\text { Passive or } \\
\text { adoptive }\end{array}$ & Antibody therapy & Lymphokine-activated NK cells \\
& Tumor-infiltrating lymphocyte & Activated T cells \\
\hline
\end{tabular}

$\mathrm{DC}=$ dendritic cell; $\mathrm{NK}=$ natural killer.

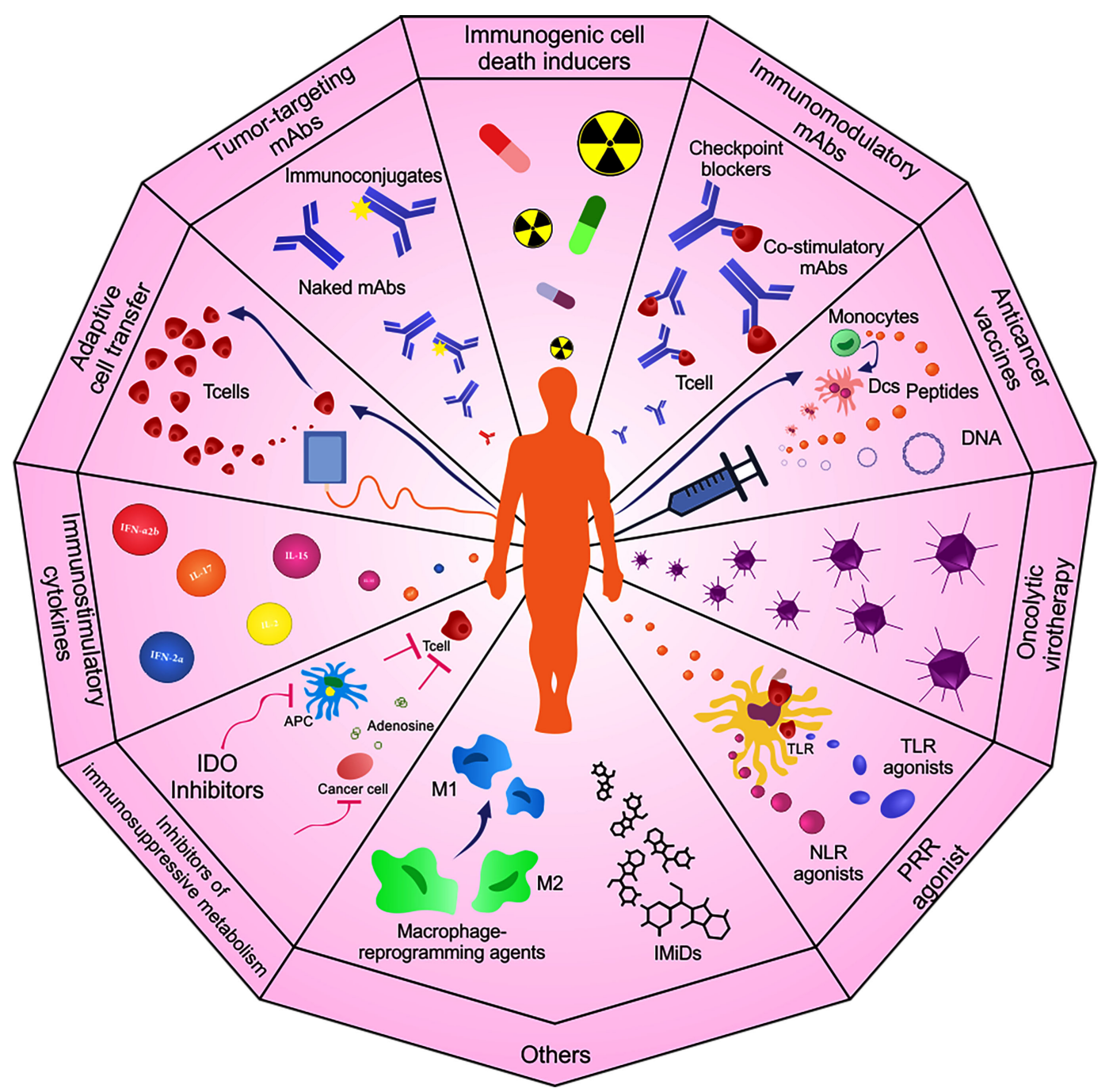

Figure 1. Anticancer immunotherapy [2]. 
There are many forms of cancer immunotherapy that have proven to be effective. The following is a summary of the design of currently common T-cell-based immunotherapies: 1) boost instruction through antigens, such as: cancer vaccines; 2) boost recognition through bypassing instruction and cell transfer, such as: adoptive T-cell therapy; 3 ) release the brakes, such as: checkpoint blockade; 4) boost instruction through bypassing presentation and cell transfer, such as: adoptive DC therapy; 5) boost recognition through bypassing instruction, cell transfer, and MHC presentation [3] [4].

\section{The Molecular Basis of Cancer Immunotherapy}

The human immune system organizes and initiates ingenuous endogenous response to foreign cells, specifically, tremendously immunogenic cancer cells, via a convoluted series of strides. These embroil performing of cancer antigens to T-cells through APCs (antigen-presenting cells), activating and priming T-cells in lymph nodes, infiltration and trafficking of T-cells towards tumor infiltrating lymphocytes (tumor beds), identification of cancer cells by T-cells, advancement of antigen-specific systemic effector and humoral immunity and memory Tcells, permitting effector T-cells, another endogenous immune cells and antibodies to tumor to pursue in concert in order to eradicate cancer cells. Usually, MHC (major histocompatibility complex) class-I APCs, for example dendritic cells (DC), introduce antigen to cluster of differentiation 8+ (CD8+) T-cells. This drives the construction of cellular cytotoxic immune response against foreign antigens. Nevertheless, this exceptionally supports sufficient antitumor immunity [5].

\section{Cancer Immunotherapeutic Approach}

One of promising treatments in cancer immunotherapeutic strategies is combined immune checkpoint (ICP) inhibitor treatment and tumor immune microenvironment (TIME)-targeted therapy. TIME appears to play an important role in tumor immune surveillance and immunological evasion, according to elevating evidence. ICP is only one of the many factors that contribute to anti-cancer immunity. Tumors utilize ICPs to defend themselves from immune system attacks. The TIME, on the other hand, is the battleground where the tumor and immune system collide, has an incalculable impact on the result of cancer immunotherapy. Accordingly, combining ICP inhibitors (ICIs) with TIME-targeting therapies is a reasonable strategy for maximizing antitumor immune response stimulation (Figure 2) [6] [7] [8] [9] [10].

\section{Cancer Immunosurveillance and Immunoediting}

Based on Merriam-Webster online dictionary

(https://www.merriam-webster.com/dictionary/immune\%20surveillance), immune surveillance is defined as: the monitoring process by which cells of the immune system (such as natural killer cells, cytotoxic T cells, or macrophages) detect and 


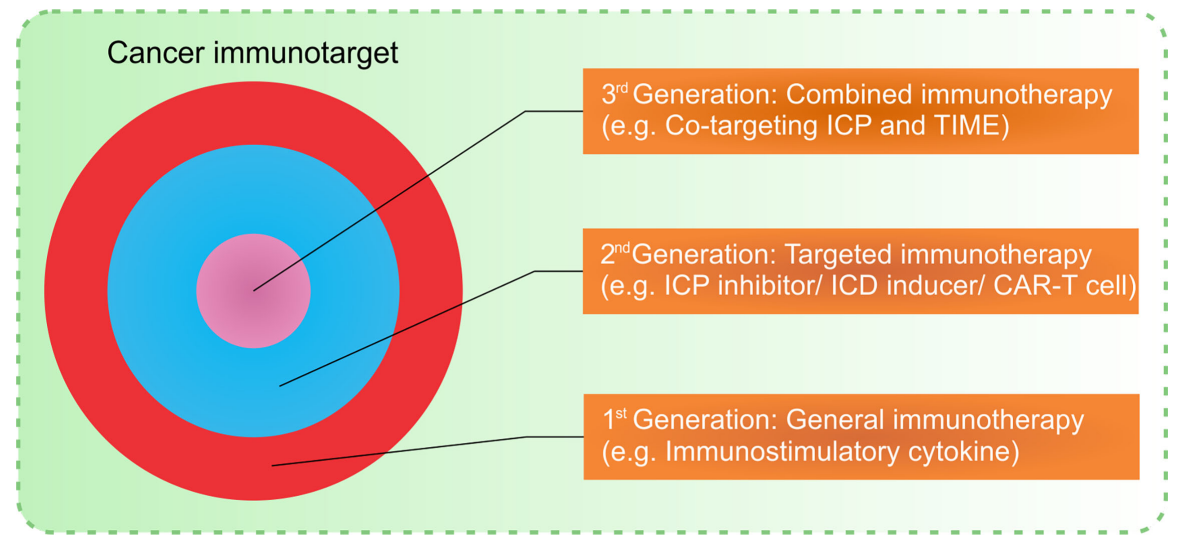

Figure 2. Development and progression of cancer immunotherapeutic strategies. The first-generation of cancer immunotherapy, which included but not definite to immunostimulatory cytokines, aimed to excite the immune system in general in order to encourage a simultaneous antitumor response. The second-generation of cancer immunotherapy, containing but not defined to CAR-T cells, immunogenic cell death (ICD) inducers, and ICP inhibitors, aimed to block peculiar immunosuppressive molecules, induce precise cellular processes, or aim specific tumor cells in order to induce a controllable antitumor response. The third generation of cancer immunotherapy, which included but was not limited to co-targeting of TIME and ICP, aimed to block various aspects of negative immune regulation at the same time in order to mount a safe and effective antitumor response [10].

destroy premalignant or malignant cells in the body. Concisely, immunosurveillance is the processes by which cells of the immune system look for and recognize foreign pathogens in the body. Immunoediting is a term coined to define the energetic interplay between a tumor and its host immune system. This process is constantly modifying the tumor's phenotype [11] [12].

Various experiments showed us that immunity can protect the host from cancer development (i.e., supplies a cancer immunosurveillance function), bolster tumor growth, sometimes by creating more aggressive tumors [13]. There is an arising awareness which cancer immunosurveillance depicts only one stride of a broader process, named cancer immunoediting, that emphasizes the tumor-sculpting actions versus dual host-protective of immune system in cancer [14].

Cancer immunoediting is an energetic process consisting of three phases (three Es): elimination, equilibrium, and escape (Figure 3). Elimination depicts the classical concept of cancer immunosurveillance. Equilibrium is the period of immunemediated latency after incomplete tumor destruction in the elimination phase. Escape recognizes the ultimate outgrowth of tumors that have surpassed immunological self-controls of the equilibrium phase [15].

Tumors persist to progress in bodies' cells with flawless immune systems despite immune surveillance.

"Cancer immunoediting" is the method by which the immune system eradicates and embodies malignant disease, and includes three stages, such as: "elimination", "equilibrium" and "escape". Elimination is the pathognomonic of the 


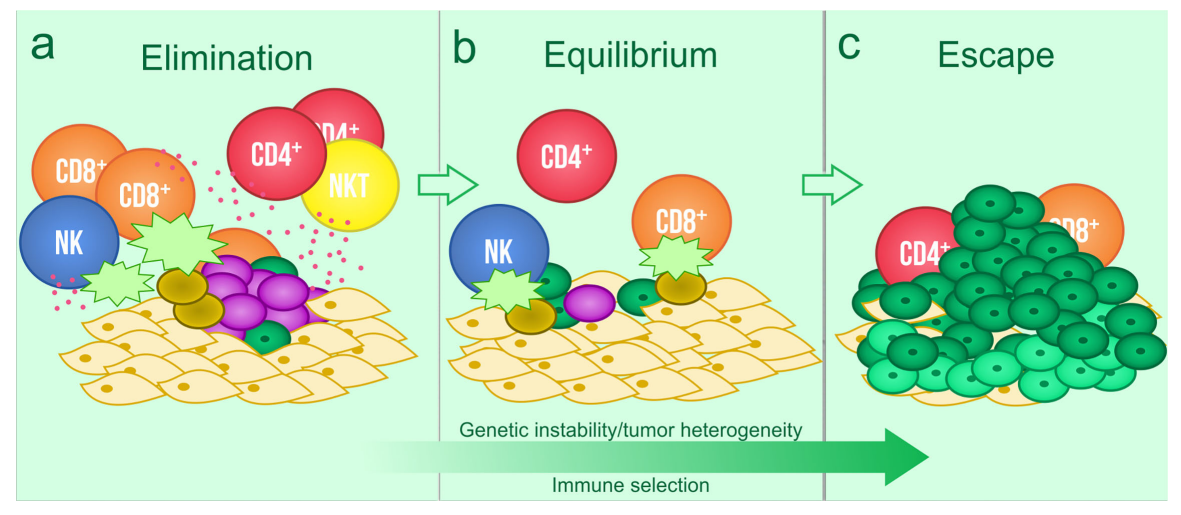

Figure 3. Three Es process of cancer immunoediting. Cancer immunoediting consists of three processes. (a) Elimination relates to immunosurveillance. (b) Equilibrium performs the process on which the immune system iteratively selects and/or promotes the generation of tumor cell variants with developing capacities to survive immune attack. (c) Escape is the mechanism wherein the immunologically chiseled tumor augments in an uncontrolled manner in the immunocompetent host. In a and b, tumor cell variants (red), underlying stroma and non-transformed cells (gray), and developing tumor cells (blue) are displayed. In c, supplementary tumor variants (orange) that have assembled as a result of the equilibrium process are demonstrated. Cytokines are shown by small orange circles. Various lymphocyte populations are as marked. The white flashes symbolize cytotoxic activity of lymphocytes against tumor cells [16].

standard idea in cancer immune surveillance by which both adaptive and innate immune responses uphold to annihilate prospering tumors. Dunn et al., recommended four aspects of elimination: 1) identification or recognition of tumor cells by innate immune cells and their definite killing, 2) maturation-migration of APCs (antigen-presenting cells) and cross-priming of T-lymphocytes, 3) creation of tumor-antigen-specific T-lymphocytes and incitement of cytotoxic systems, and 4) homing of tumor-antigen-specific T-lymphocytes to the tumor area and eradication of tumor cells [14] [15] [16].

This is pursued by the serenity phase which comprises endless designing of tumor cells and choice of those with diminished immunogenicity, bolstering the result of impervious variants. Cancer cells can invade repose or a slow-cycling condition and endure hidden for continued periods of time. Novel variants with various mutations that escalate resistance to immune pressure arise as long as the equilibrium process, the longest of the three phases that lasts several years [17] [18].

\section{The Hallmarks of Cancer (Can Be) Revisited}

The enormous inventory of cancer cell genotypes is a representation of six important changes in cell physiology which systematically govern malignant growth (Figure 4): apoptosis evasion, sustained angiogenesis, self-sufficiency in growth signals, insensitivity to growth-inhibitory (antigrowth) signals, tissue invasion and metastasis, and unlimited replicative capacity [19].

The six cancer hallmarks-extraordinary and interdependent competences that allow tumor development and metastasis-endure to support a solid foun- 
dation for comprehension about cancer biology (Figure 5) [20].

The hallmarks of cancer have been reinterpreted as seven hallmarks, such as: a facilitating microenvironment, immune modulation, modified stress response encouraging overall survival, metabolic rewiring, selective growth and proliferative benefit, vascularization, invasion and metastasis (Figure 6). Albeit, the concept of the hallmarks implies similarity, it is crucial to understand that cancer is not a single disease, and that tumor types vary in their reliance on-and-off effects of shared pathways. For instance, while vascularization is an essential cancer hallmark, some tumors are weakly vascular and can depend less on it [21] [22].

\section{Nanotechnology Based Cancer Immunotherapy}

With the advent of nanotechnology, precise, safer, and more efficient cancer

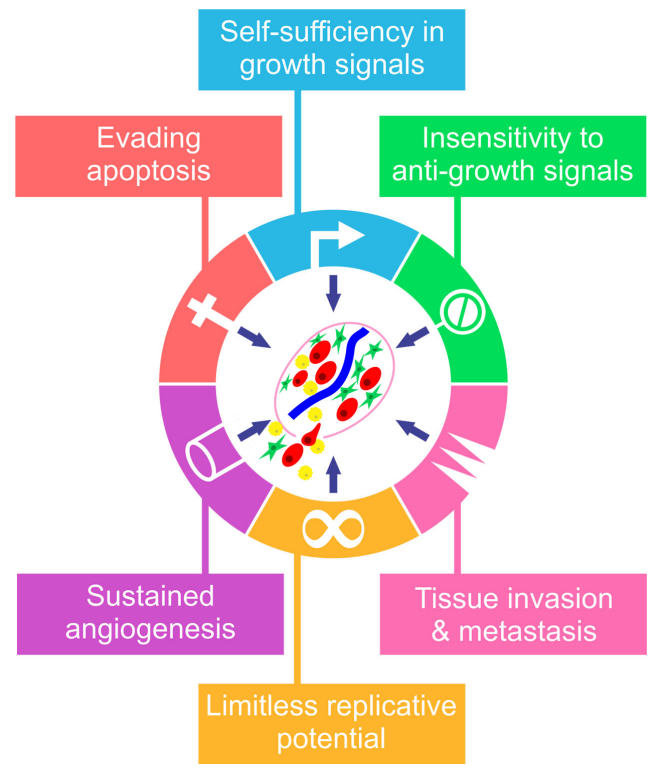

Figure 4. Acquired Capabilities of Cancer [19].

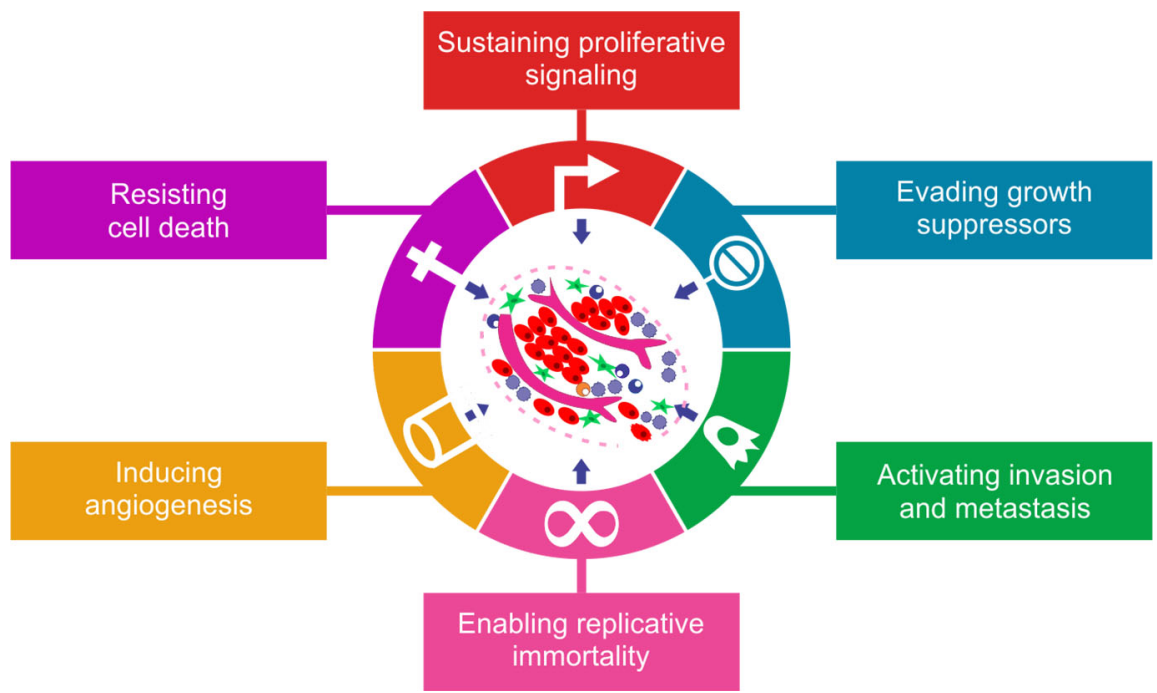

Figure 5. The hallmarks of cancer [20]. 


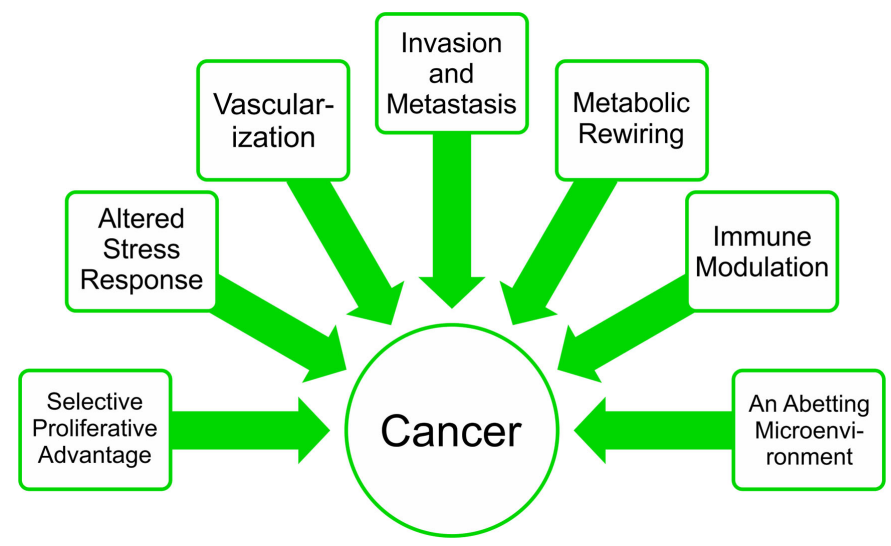

Figure 6. The hallmarks of cancer revisited [21].

immunotherapy is becoming a reality. The versatility to tune biodistribution, biocompatibility, immunogenicity, minimal degradation of bioactive molecules, regulated loading, precise targeting, sustained release kinetics, controlled spatiotemporal delivery profiles together with adjuvants has grasped the nanoparticles (NPs) based therapy to arise as an efficient method [23] [24].

The nanoscale size and versatility have helped NPs increase the effectiveness of conventional cancer immunotherapy and opened up exhilarating ways to fight cancer. The use of NP-based methods has aided in improving the effectiveness of traditional cancer vaccines and overcoming tumor microenvironment (TME)related challenges. There are various strategies about NPs mediated immunomodulation of TME. They are: 1) NPs mediated dissolution of ECM; 2) NPs targeting vasculature; 3) NPs targeting cytokines in TME; 4) NPs mediated depletion of cancer associated fibroblast (CAF); 5) NPs mediated reprogramming of TAM; 6) NPs mediated suppression of Treg; 7) NPs mediated modulation of myeloid derived stem cells (Figure 7) [25].

\section{Immunovaccinomics}

The new era of vaccines was started in 1774. At that time, Benjamin Jesty, a farmer, observed a milkmaid who had cowpox, but did not have smallpox. He inoculated his two sons and his wife. This was done 22 years, long before the inoculation process and publication carried out by Edward Jenner in 1798. It was only in the 19th century that the vaccinia virus (poxvirus in mice) proved to be effective in replacing cowpox as a vaccine [26] [27].

Experts first used the smallpox virus as a vaccine candidate until the 1990s. Vaccine development in that era was still at an empirical stage, with an old perspective, namely: isolation, inactivation, and injection. The fast pace of technology and information encourages the making of vaccines to progress [28].

The progress of the second generation of hepatitis $B$ vaccine is now based on molecular medicine. The next era of vaccines was brought on by the activation of the immune mechanism through the utilization and conjugation of proteins (pneumococcal and meningococcal vaccines), and the HPV vaccine. 


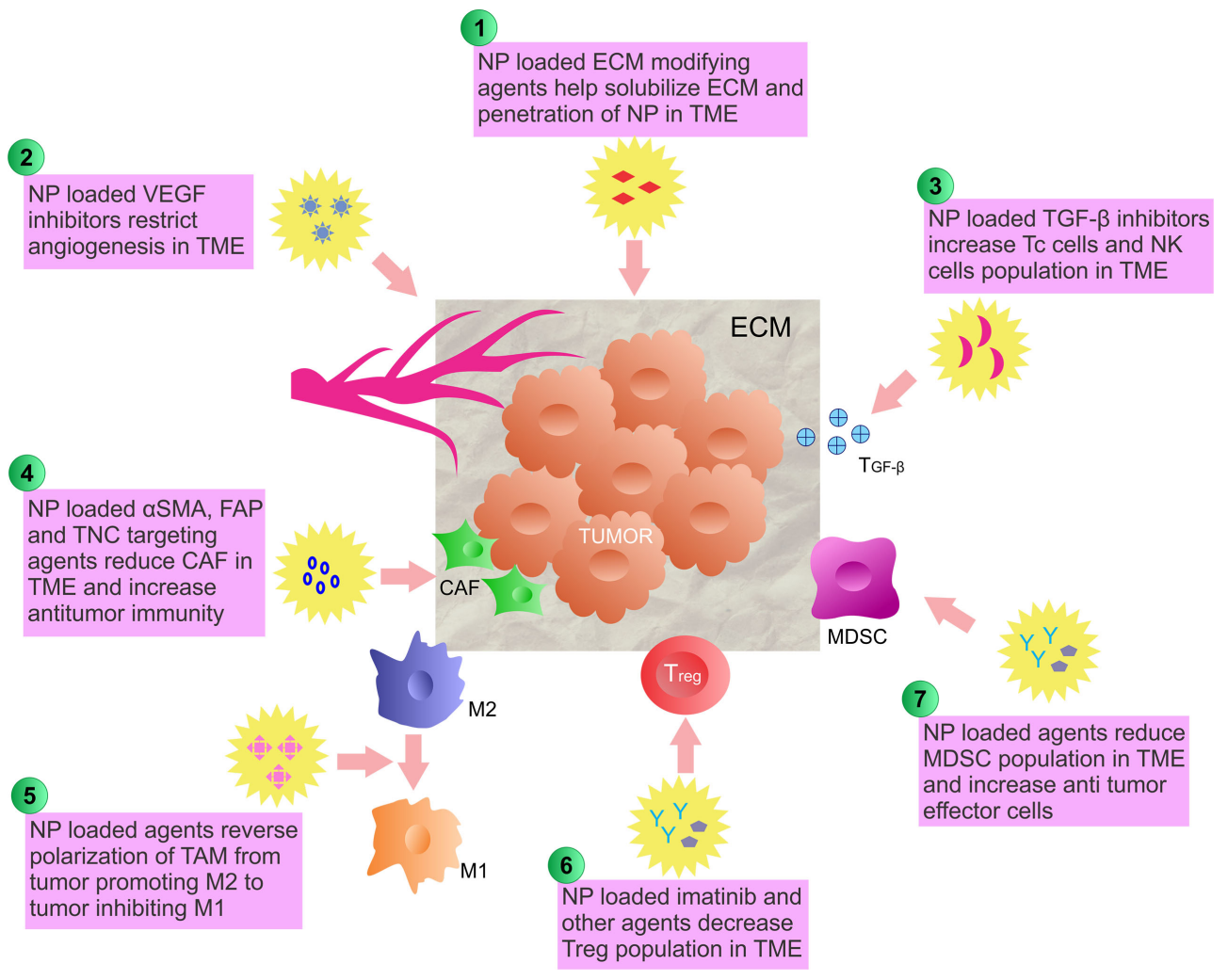

Figure 7. NPs mediated TME modulation for effective immunotherapy. NPs are used to modify the TME by various approaches to enhance antitumor immune response [25].

New perspectives based on immunogenomics and the sophistication of highdimensional genetic and immunological assays facilities, in the form of whole genome sequences, transcriptomic-based mRNA approaches, the latest bioinformatic approaches to study the complexity of immune system mechanisms have made vaccinology enter a new era, namely vaccinomics [29] [30] [31].

\section{Vaccinomics Approach}

Vaccinomics is a multifaceted scientific field that studies immunological and biological vaccine responses, as well as the heterogeneity of immune responses. To design logical vaccine approaches, detailed research on the processes underlying host responses to pathogens is combined with high-dimensional epigenetic, genetic, transcriptomic, and proteomic analyses, which culminate in protective immunity. These insights lead to the discovery of new knowledge, which is then applied to the creation of new vaccine candidates after replication and validation, resulting in new insights into biological processes that further scientific knowledge and discovery [32] [33] [34].

Immunogenetics and immunogenomics are integrated with biological systems and immune profiling in vaccinomics. For the production of next generation vaccines and the development of scientists' capabilities in the field of individualized medicine, vaccinomics is focused on the use of high-tech assays (based on omics) and the current bioinformatics approaches. 
The study was based on genotypic-phenotypic data, supported by comprehensive immune response mechanism milestones that "force" scientists to innovate and uncover the veils behind the interactions between the genes on the heterogeneity of the immune response, as well as the effects of genetic polymorphisms on vaccines. This requires a comprehensive data bank, in the form of genotypic data such as: transcriptomics, gene sequences. Phenotypic data, for both vaccine responders and non-responders, is also needed. Large data is needed by scientists to carry out the process of analysis, observation, replication, interpretation, validation, and implementation.

Sophisticated bioinformatics-based facilities and infrastructure can be utilized to develop and detect immune response profiles to understand vaccine development and usage. The recent era of omics-based technology (metabolomics, proteomics, pharmacogenomics, nutrigenomics, etc.) and personalized medicine has acculturated with vaccinology, revolutionizing pharmacology and therapy. It has welcomed the era of personal vaccinology, which is predictive and futuristic.

The development of vaccinomics mustpay attention to the initial purpose of vaccination in order to activate and extend the protection of various antigen-specific $\mathrm{B}$ and $\mathrm{T}$ cells in eliminating the infection process or the availability of sufficient antigens for protection.

Personalized medicine (PM) has a similar principle to personalized vaccinology (PV). That is, regulations for choosing the right vaccine need consideration of genetic and individual characteristics. In other words, the administration of vaccines needs rational considerations, namely: the right individual, the right vaccine, the right time, the right dose, the right targets/aims (metabolism, genetics, etc.). The problem is, not everyone responds to vaccines the same way [35].

Optimal PV perspectives include adjuvants, vaccine formulation, dosage, route of administration, schedule of administration, especially for vaccines with multiple doses for groups or individuals. The application of vaccinomics helps scientists and the public to understand the diversity of genetic and non-genetic factors that influence immune mechanisms and vaccine antigens in various organ systems. Scientists in the vaccinomics era also need to pay attention to the effectiveness of vaccines that are determined to be multifactorial. For example, replication, activation, and differentiation of $\mathrm{T}$ and $\mathrm{B}$ lymphocytes trigger the generation of memory cells.

\section{The Theory of Vaccinology}

Poland et al. (2016), [36] explained that there are various perspectives, approaches, studies, models, and the latest theories that discuss vaccinology. This multiparadigm explains the various current theories or models that are implemented in understanding vaccine-induced immune responses and the development of vaccine quantity-qualities.

First, the reverse vaccinology model. This model is based on genomic data and uses in silico analysis to accelerate the identification of antigens in the vaccine 
development process. This model uses transcriptomics, proteomics, epitope prediction algorithms, and immune monitoring as "tools".

Second, the immune response network theory. This theory explains immunity as a predictive resultant through the activation and sequential interactions of various genes and various gene pathways. This theory uses "tools" in the form of transcriptomics, proteomics, and pathways analysis.

Third, vaccinomics. This theory discusses a comprehensive study of the immune system, response and mechanism to vaccination so that scientists can understand and predict vaccine-induced immunity, then immediately apply it to the innovation and development of vaccines rationally. This theory uses "tools" in the form of epigenomic, proteomic, immunogenetic/immunogenomic, transcriptomic, immune system monitoring, and computational modeling.

Fourth, systems vaccinology theory. This theory explains the application of various biological methods and systems to enable scientists to comprehensively predict and understand vaccine-inducing immune responses. This theory uses "tools" in the form of transcriptomic, proteomic, epigenic, and computational modeling.

Fifth, structural vaccinology theory. This theory explains the utilization of various structural biology studies to facilitate the selection of vaccine epitopes. The equipment used by this model is based on nuclear magnetic resonance (NMR), proteomics, X-ray crystallography, and immune system surveillance.

Sixth, informatic vaccine theory. This theory explains the competence of bioinformatics to facilitate vaccine production, licensing, development, and testing. The tools used by this approach are based on epitope prediction algorithms, computational modeling, HLA-binding algorithms, mathematical simulation of immune responses, and integrated data mining.

The lesson from the application of genomics to the world of vaccinology is that not all side effects or vaccine reactions are directly related to vaccines. According to Verbeek et al. (2014), the development of epilepsy after compulsory immunization for children is caused by a combination of events, genetic or structural defects [37]. During the first two years of life, scientists studied 990 children who had postimmunization seizures (1 dose of MMR, 4 doses of DTaP, Haemophilus influenza type B vaccine).

The inactivated vaccine and the live attenuated vaccine caused 68 percent and 32 percent of the 1022 possible epileptic seizures among these 990 children, respectively [38].

\section{Adversomics}

Vaccinomics is an adversomic's "best friend". The two of them are inseparable. The term adversomics emerged in 2009, referring to the understanding of side effects or vaccine reactions based on biological and immunogenomic systems. Simply put, adversomics is a new perspective in vaccine design and safety. Scientists and clinicians urgently need an understanding of basic science, including 
immunology, molecular biology, and translational, supported by clinical integration to gain a comprehensive understanding of the mechanisms of vaccine side effects in order to design new vaccines that eliminate various pathological events and infection, while avoiding all possible side effects.

Vaccine adversomic studies are an extension of pharmacogenomic studies. The difference is, if pharmacogenomics studies drugs based on genomic aspects, then adversomics understands vaccines based on a genomic perspective. The methodology of both is relatively the same, although the exact mechanism causing the reaction or side effect of the vaccine is not known until now. Deepening cross-sectional/multidisciplinary involvement to the basics, covering the genetic, molecular, proteomic, adversomic aspects, in particular how the genetic aspects (i.e. genomics and transcriptomics) have a strong effect on the development of vaccine side effects and side reactions, as well as providing insight in the process of designing new safer and more effective vaccine candidates

Scientists' understanding of adversomics is broadened by the contribution of the HLA gene to vaccine-inducing immune mechanisms and responses, as well as by side effects. Implementation of phenotype and genotype studies is important in innovating models of genetic predisposition and vaccine side effects.

Understanding vaccinomics through the immunogenetic-genomic paradigm can be applied to study and accelerate vaccine-response mechanisms. Omic-based technologies, including vaccinomics, have evolved rapidly beyond host genetic variation, with functional effects (from an epigenic, proteomic, transcriptomic perspective) through these variations. Collaboration, synergy and research harmonization are needed among experts, scientists, researchers, clinicians for the development of vaccinomics in the future [39] [40].

\section{Immunogenomic Perspectives}

In recent years, there has been an explosion of information about innate immunity components and their function in guiding and shaping the adaptive immune response. The comprehension of PRR (pathogen recognition receptors), especially the TLR (Toll-like receptors) family, as well as associated pathways and their role in host responses when exposed to foreign antigens in the form of vaccines or pathogens, has exploded [41] [42] [43] [44]. Many new patterns have been added to the mosaic, explaining how innate immune system cells process and identify antigens, as well as how this affects the duration (immune memory) of B-cell immune responses and adaptive $\mathrm{T}$ cells naturally.

Live attenuated vaccines (LAVs) carry native pathogen-associated molecular signals, i.e., viral genetic material that activates the innate immune system via PRRs (pathogen recognition receptors). The LAVs multiply and join the host immune system, where they are picked up by dendritic cells (DCs) or antigenpresenting cells (APCs), which then move to lymphoid organs to demonstrate antigens to $\mathrm{T}$ and $\mathrm{B}$ lymphocytes. This triggers the same immune responses as natural infections, and it's usually successful after only one dose. These vaccines, 
however, can cause mild to severe side effects in patients due to minimal replication at the host [44] [45].

Biological systems use a method that is both particular and systematic. It has the potential to not only accelerate the production of new innate immunity regulators, but also to provide a thorough understanding of kinetic regulation at the transcriptional, interprotein, and post-transcriptional levels. All of this knowledge will help to make vaccine discoveries "high impact," by providing molecular predictive indicators of vaccine immunogenicity, opening up new vaccine efficacy correlations, and assisting in the creation of novel vaccine antigens or formulations. Furthermore, the system-level approach allows for the detection of responders and non-responders, resulting in improved immunological coverage for approved vaccines. Identification of transcriptional gene profiles, and more broadly, immune signatures relevant to vaccine immune responses, will serve as the foundation for tailored care to achieve the best clinical outcome [44] [46].

Vaccines are the most practical public health appliances for governing infectious diseases. There are a large number of novel and re-emerging pathogens for which we do not have adequate vaccines and there is area for advancement in various contemporary vaccines, regardless of extensive success. Vaccine improvement encounters a number of hurdles, many of which are revealed here (Figure 8). Establishing vaccines to resist prevailing and impending or prospective pathogens will need us to surmount those obstacles and current progress in genomic sciences may support the solutions that we desire [47].

The high-dimensional assays and advanced genetic, supported by bioinformatics technologies have experienced to a contemporary era of genetic arrangement of vaccines and have contributed real solutions to the impediments presently hindering advancement in this scope (Table 2) [47].

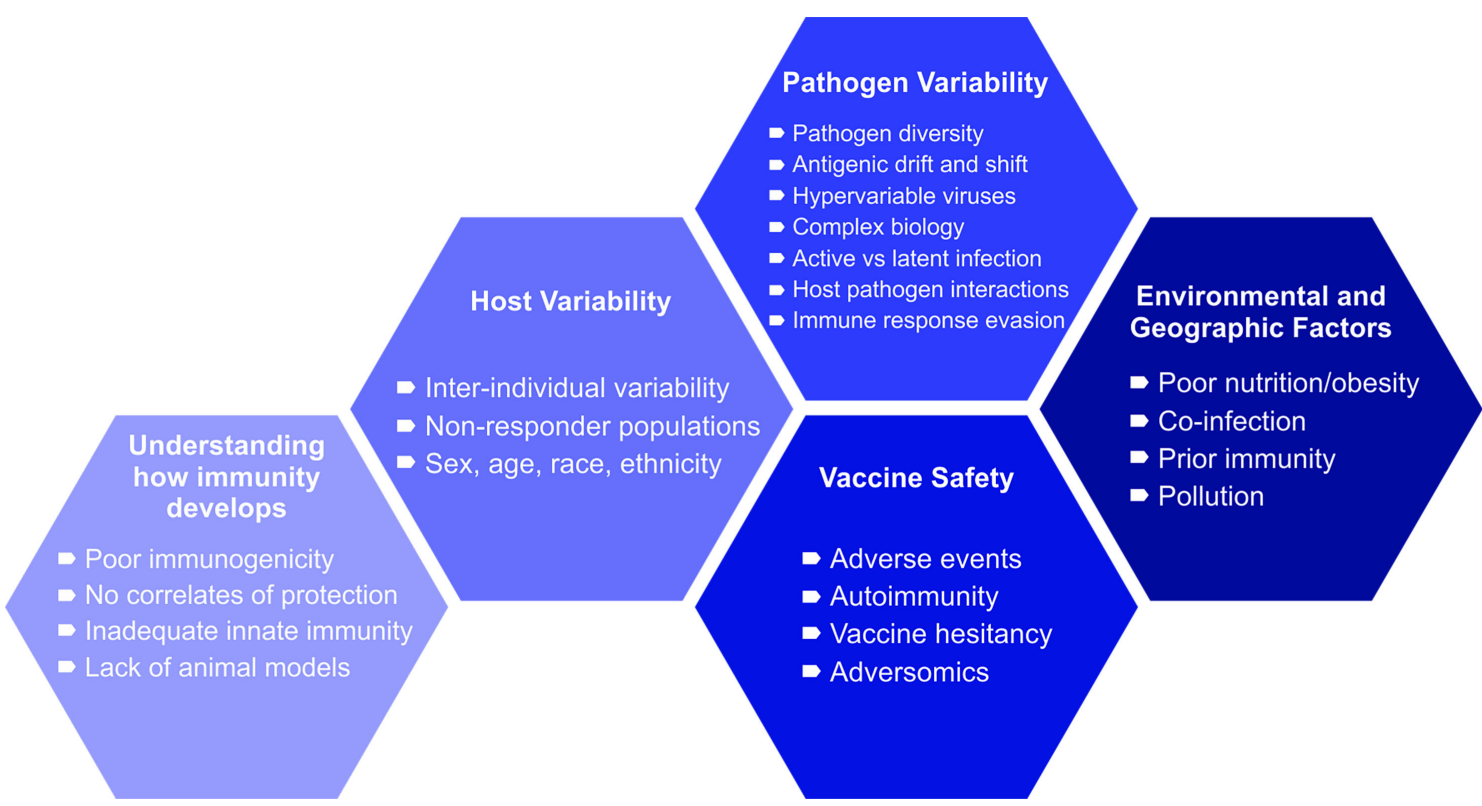

Figure 8. Barriers to vaccine development [47]. 
Table 2. Genomics-based solutions to vaccine development barriers [47].

\begin{tabular}{ll}
\hline Barrier & Potential solution(s) \\
\hline $\begin{array}{l}\text { Understanding how } \\
\text { immunity develops }\end{array}$ & \\
- Poor immunogenicity & Systems biology studies \\
and/or durability & Identify non-humoral correlates of protection \\
- Lack of correlates of & Better understanding of the effector functions associated \\
protection & with spontaneous resolution of infection \\
- Inefficient activation of & Vaccines inducing cellular immunity \\
innate immunity & Laboratory assays measuring functional responses \\
- Lack of animal models with & correlated with clinical protection \\
predictive value &
\end{tabular}

\section{Host variability}

- Inter-individual variability Age, sex, or population-based vaccine formulations in vaccine response Diagnostic tests to predict vaccine response

- Non-responder populations

- Sex, age, race, ethnic differences in response

\section{Pathogen variability}

- Pathogen diversity

Vaccines eliciting broadly neutralizing Abs

- Antigenic drift and antigenicMulti-valent vaccines eliciting high affinity Ab to multiple shift serotypes

- Hypervariable viruses Universal vaccine based on genetically conserved epitopes

- Complex biology (e.g., Plasmodium) Vaccines targeting pre-erythrocytic, blood, and/or

- Active vs. latent vs. chronic Interventions that mitigate pathogen immunomodulation infection during immune response to vaccination

- Host pathogen interactions DNA vaccine targeting $\mathrm{T}$ cell responses to the partially

- Immune response evasion conserved NS3 and $\mathrm{C}$ genes and $\mathrm{Ab}$ responses to the $\mathrm{E}$ mutants protein

\section{Vaccine safety}

- Adverse events

- Autoimmunity

- Vaccine hesitancy

Subunit, protein, and peptide-based vaccines incorporating novel adjuvants driving immunogenicity and durable protection Dose-sparing approaches

\section{Environmental and} geographic factors

- Poor nutrition/obesity

DENV-vaccines for naïve and DENV-exposed individuals

- Co-infection

- Prior immunity Vitamin supplementation coadministered with

- Pollution vaccination

With the latest developments in technology-based multidisciplinary omics, personalized medicine-based vaccines will be better developed, of course still paying attention to safety, efficacy, and (bio) ethical aspects [48] [49]. 


\section{Acknowledgements}

We thank Dr. Andrew San Antonio for critical reading of our manuscript.

\section{Conflict of Interest Statement}

The authors declare that the research was conducted in the absence of any commercial or financial relationships that could be construed as a potential conflict of interest. Authors claim no conflicts of interest related to the research described in the manuscript.

\section{References}

[1] Schrama, D., Reisfeld, R.A. and Becker, J.C. (2006) Antibody Targeted Drugs as Cancer Therapeutics. Nature Reviews Drug Discovery, 5, 147-159. https://doi.org/10.1038/nrd1957

[2] Galluzzi, L., et al. (2014) Classification of Current Anticancer Immunotherapies. Oncotarget, 5, 12472.

[3] Tokuyasu, T.A. and Huang, J.-D. (2018) A Primer on Recent Developments in Cancer Immunotherapy, with a Focus on Neoantigen Vaccines. Journal of Cancer Metastasis and Treatment, 4, 2. https://doi.org/10.20517/2394-4722.2017.52

[4] Schirrmacher, V. (2019) From Chemotherapy to Biological Therapy: A Review of Novel Concepts to Reduce the Side Effects of Systemic Cancer Treatment. International Journal of Oncology, 54, 407-419.

[5] Sukari, A., et al. (2016) Cancer Immunology and Immunotherapy. Anticancer Research, 36, 5593-5606. https://doi.org/10.21873/anticanres.11144

[6] Binnewies, M., et al. (2018) Understanding the Tumor Immune Microenvironment (TIME) for Effective Therapy. Nature Medicine, 24, 541-550.

https://doi.org/10.1038/s41591-018-0014-x

[7] Nagarsheth, N., Wicha, M.S. and Zou, W. (2017) Chemokines in the Cancer Microenvironment and Their Relevance in Cancer Immunotherapy. Nature Reviews Immunology, 17, 559. https://doi.org/10.1038/nri.2017.49

[8] Zhang, Y. and Chen, L. (2016) Classification of Advanced Human Cancers Based on Tumor Immunity in the MicroEnvironment (TIME) for Cancer Immunotherapy. JAMA Oncology, 2, 1403-1404. https://doi.org/10.1001/jamaoncol.2016.2450

[9] Chen, D.S. and Mellman, I. (2017) Elements of Cancer Immunity and the CancerImmune Set Point. Nature, 541, 321-330. https://doi.org/10.1038/nature21349

[10] Tang, T., et al. (2021) Advantages of Targeting the Tumor Immune Microenvironment over Blocking Immune Checkpoint in Cancer Immunotherapy. Signal Transduction and Targeted Therapy, 6, 1-13. https://doi.org/10.1038/s41392-020-00449-4

[11] Teng, M.W., et al. (2015) From Mice to Humans: Developments in Cancer Immunoediting. The Journal of Clinical Investigation, 125, 3338-3346. https://doi.org/10.1172/JCI80004

[12] Zhang, A.W., et al. (2018) Interfaces of Malignant and Immunologic Clonal Dynamics in Ovarian Cancer. Cell, 173, 1755-1769.e22.

[13] Smyth, M.J., Dunn, G.P. and Schreiber, R.D. (2006) Cancer Immunosurveillance and Immunoediting: The Roles of Immunity in Suppressing Tumor Development and Shaping Tumor Immunogenicity. Advances in Immunology, 90, 1-50. https://doi.org/10.1016/S0065-2776(06)90001-7 
[14] Dunn, G.P., Old, L.J. and Schreiber, R.D. (2004) The Immunobiology of Cancer Immunosurveillance and Immunoediting. Immunity, 21, 137-148. https://doi.org/10.1016/j.immuni.2004.07.017

[15] Dunn, G.P., Old, L.J. and Schreiber, R.D. (2004) The Three Es of Cancer Immunoediting. Annual Review of Immunology, 22, 329-360. https://doi.org/10.1146/annurev.immunol.22.012703.104803

[16] Dunn, G.P., et al. (2002) Cancer Immunoediting: From Immunosurveillance to Tumor Escape. Nature Immunology, 3, 991-998. https://doi.org/10.1038/ni1102-991

[17] Malladi, S., et al. (2016) Metastatic Latency and Immune Evasion through Autocrine Inhibition of WNT. Cell, 165, 45-60. https://doi.org/10.1016/j.cell.2016.02.025

[18] Baxevanis, C.N. and Perez, S.A. (2015) Cancer Dormancy: A Regulatory Role for Endogenous Immunity in Establishing and Maintaining the Tumor Dormant State. Vaccines, 3, 597-619. https://doi.org/10.3390/vaccines3030597

[19] Hanahan, D. and Weinberg, R.A. (2000) The Hallmarks of Cancer. Cell, 100, 57-70. https://doi.org/10.1016/S0092-8674(00)81683-9

[20] Hanahan, D. and Weinberg, R.A. (2011) Hallmarks of Cancer: The Next Generation. Cell, 144, 646-674. https://doi.org/10.1016/j.cell.2011.02.013

[21] Fouad, Y.A. and Aanei, C. (2017) Revisiting the Hallmarks of Cancer. American Journal of Cancer Research, 7, 1016.

[22] Carmeliet, P. and Jain, R.K. (2000) Angiogenesis in Cancer and Other Diseases. Nature, 407, 249-257. https://doi.org/10.1038/35025220

[23] Raju, G.S.R., et al. (2019) Nanomaterials Multifunctional Behavior for Enlightened Cancer Therapeutics. Seminars in Cancer Biology, 69, 178-189.

[24] Koshy, S.T. and Mooney, D.J. (2016) Biomaterials for Enhancing Anti-Cancer Immunity. Current Opinion in Biotechnology, 40, 1-8.

https://doi.org/10.1016/j.copbio.2016.02.001

[25] Gupta, J., Safdari, H.A. and Hoque, M. (2021) Nanoparticle Mediated Cancer Immunotherapy. Seminars in Cancer Biology, 69, 307-324.

https://doi.org/10.1016/j.semcancer.2020.03.015

[26] Pead, P.J. (2014) The Origins of Vaccination: History Is What You Remember. Journal of the Royal Society of Medicine, 107, 7. https://doi.org/10.1177/0141076813513998

[27] Hammarsten, J.F., Tattersall, W. and Hammarsten, J. (1979) Who Discovered Smallpox Vaccination? Edward Jenner or Benjamin Jesty? Transactions of the American Clinical and Climatological Association, 90, 44.

[28] Plotkin, S.L. and Plotkin, S.A. (2004) A Short History of Vaccination. Vaccines, 5, 1-16. https://doi.org/10.1016/B978-1-4160-3611-1.50005-2

[29] Chen, Z., et al. (2021) Identification of COVID-19 Subtypes Based on Immunogenomic Profiling. International Immunopharmacology, 96, Article ID: 107615. https://doi.org/10.1016/j.intimp.2021.107615

[30] Wang, Q., et al. (2021) Immunogenomic Identification for Predicting the Prognosis of Cervical Cancer Patients. International Journal of Molecular Sciences, 22, 2442. https://doi.org/10.3390/ijms22052442

[31] Higdon, R., et al. (2017) Integrated Proteomic and Transcriptomic-Based Approaches to Identifying Signature Biomarkers and Pathways for Elucidation of Daoy and UW228 Subtypes. Proteomes, 5, 5. https://doi.org/10.3390/proteomes5010005

[32] Haralambieva, I.H. and Poland, G.A. (2010) Vaccinomics, Predictive Vaccinology and the Future of Vaccine Development. Future Microbiology, 5, 1757-1760. 
https://doi.org/10.2217/fmb.10.146

[33] Omersel, J. and Karas Kuželički, N. (2020) Vaccinomics and Adversomics in the Era of Precision Medicine: A Review Based on HBV, MMR, HPV, and COVID-19 Vaccines. Journal of Clinical Medicine, 9, 3561. https://doi.org/10.3390/jcm9113561

[34] de la Fuente, J., et al. (2020) A Quantum Vaccinomics Approach to Vaccine Development. The Cuban Scientist, 1, 25-26.

[35] Poland, G.A., Ovsyannikova, I.G. and Kennedy, R.B. (2018) Personalized Vaccinology: A Review. Vaccine, 36, 5350-5357.

https://doi.org/10.1016/j.vaccine.2017.07.062

[36] Poland, G.A., et al. (2016) Vaccinology in the Third Millennium: Scientific and Social Challenges. Current Opinion in Virology, 17, 116-125. https://doi.org/10.1016/j.coviro.2016.03.003

[37] Verbeek, N.E., et al. (2014) Etiologies for Seizures around the Time of Vaccination. Pediatrics, 134, 658-666. https://doi.org/10.1542/peds.2014-0690

[38] Li, X., et al. (2018) The Influence of Vaccine on Febrile Seizure. Current Neuropharmacology, 16, 59-65. https://doi.org/10.2174/1570159X15666170726115639

[39] Trein, P. and Wagner, J. (2021) Governing Personalized Health: A Scoping Review. Frontiers in Genetics, 12, 513. https://doi.org/10.3389/fgene.2021.650504

[40] Gerber, J.E. (2020) Characterization of Current and Future Vaccination Challenges in the United States: Influenza and the Policy Implications of Vaccinomics. Johns Hopkins University, Baltimore.

[41] Kawai, T. and Akira, S. (2010) The Role of Pattern-Recognition Receptors in Innate Immunity: Update on Toll-Like Receptors. Nature Immunology, 11, 373. https://doi.org/10.1038/ni.1863

[42] Olive, C. (2012) Pattern Recognition Receptors: Sentinels in Innate Immunity and Targets of New Vaccine Adjuvants. Expert Review of Vaccines, 11, 237-256. https://doi.org/10.1586/erv.11.189

[43] Werling, D., Piercy, J. and Coffey, T.J. (2006) Expression of TOLL-Like Receptors (TLR) by Bovine Antigen-Presenting Cells-Potential Role in Pathogen Discrimination? Veterinary Immunology and Immunopathology, 112, 2-11. https://doi.org/10.1016/j.vetimm.2006.03.007

[44] Buonaguro, L. and Pulendran, B. (2011) Immunogenomics and Systems Biology of Vaccines. Immunological Reviews, 239, 197-208. https://doi.org/10.1111/j.1600-065X.2010.00971.X

[45] Bose, D. (2017) Tat-Independent Lentivirus Genomes for Vaccination and Host/ Pathogen Interaction Studies. Université Grenoble Alpes, Saint-Martin-d'Hères.

[46] Kreileder, M., et al. (2020) Signaling Dynamics Regulating Crosstalks between TCell Activation and Immune Checkpoints. Trends in Cell Biology, 31, 224-235. https://doi.org/10.1016/j.tcb.2020.12.001

[47] Kennedy, R.B., et al. (2020) Current Challenges in Vaccinology. Frontiers in Immunology, 11, 1181. https://doi.org/10.3389/fimmu.2020.01181

[48] Gerber, J.E., et al. (2021) Ethical and Policy Implications of Vaccinomics in the United States: Community Members' Perspectives. Human Vaccines \& Immunotherapeutics, 1-12.

[49] Anurogo, D., Parikesit A.D. and Ikrar, T. (2019) LncRNAs in CONDBITs Perspectives, from Genetics towards Theranostics (LncRNAs Dalam Perspektif CONDBITs, Dari Genetik ke Theranostik). Malaysian Journal of Health Sciences/Jurnal Sains Kesihatan Malaysia, 17, 1-16. https://doi.org/10.17576/jskm-2019-1702-01 